\title{
Computer Generation of Ribbed Sculptures
}

\author{
James Hamlin and Carlo H. Séquin ${ }^{1}$ \\ CS Division, University of California, Berkeley \\ E-mail: sequin@cs.berkeley.edu
}

\begin{abstract}
Charles Perry's monumental sculpture Solstice is analyzed and its generative geometrical logic based on a twisted toroidal sweep is captured in a computer program with interactively adjustable control parameters. This program is then used to generate other models of ribbed sculptures based on one or more interlinked torus knots. From this family of sculptures related to Perry's Solstice we derive a broader paradigm for the generation of "ribbed" sculptures. It is based on one or two simple, mathematically defined "guide rails," which are then populated with a dense set of thinner "ribs" to create lightweight, transparent surfaces. With this broadened concept and a few suitably modified and parameterized programs we can emulate many other ribbed sculptures by Charles Perry and also create new sculpture designs and mathematical visualization models that profit from the semi-transparent look of these structures.
\end{abstract}

Keywords: ribbed sculpture; computer generated geometry; sweep surfaces; Charles Perry.

AMS Subject Classification: 68N99; 51M99
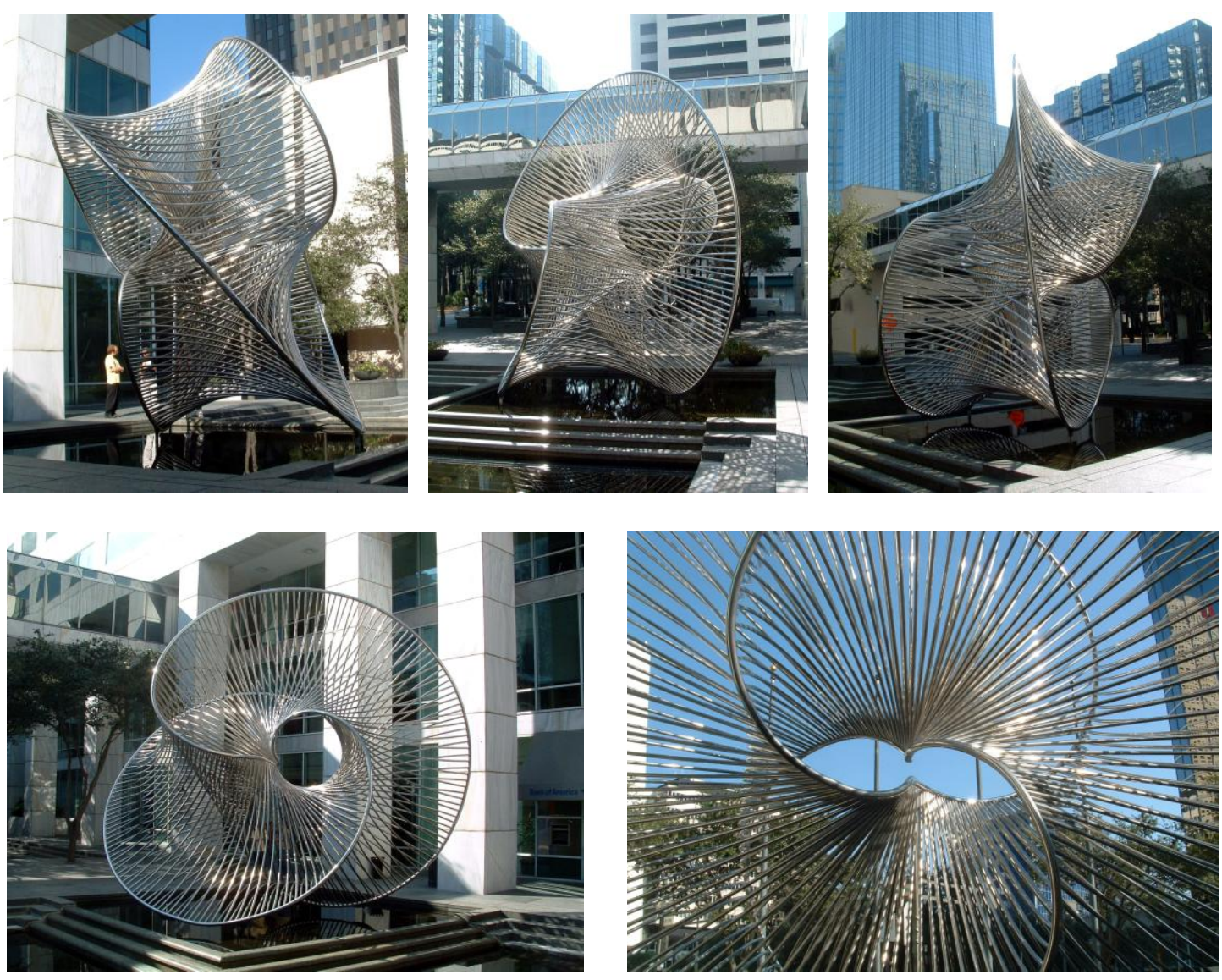

Figure 1. Five different views of Charles Perry's Solstice in downtown Tampa, Florida (1985)

[Photos copyright C. H. Séquin, 2007]

\footnotetext{
1 Author Posting. (C) Taylor \& Francis, 2010. This is the author's version of the work. It is posted here by permission of Taylor \& Francis for personal use, not for redistribution. The definitive version was published in Journal of Mathematics and the Arts, Volume 4 Issue 4 , December 2010. doi:10.1080/17513472.2010.487183 (http://dx.doi.org/10.1080/17513472.2010.487183)
} 


\section{Introduction}

The 28-foot tall Solstice sculpture by Charles Perry (Figure 1), located in downtown Tampa, Florida is a prime example of the "ribbed sculptures" to be discussed here. Ribbed sculptures offer a translucent, "airy" presence in indoor as well as outdoor settings. Because of the substantial open space between the ribs, they do not cast harsh shadows or block views completely. Moreover, they are reasonably cost effective to be constructed at a large scale - much less expensive than large free-form bronze sculptures, investment cast from many individual molds.

These ribbed sculptures may trace their roots to the pioneering work of some constructivist artists as well as to the mathematical string models of conic and bilinear surfaces that one can find in science museums. The ribbed approach to defining a shape in space is particularly valid and economical at an architectural scale, as it makes use of Naum Gabo's vision that space could be represented without having to employ a lot of mass. Gabo demonstrated this principle with more than two dozen versions of his Linear Construction made of nylon monofilament strung over transparent plastic frames. Serious artists often implement many versions of some worthwhile concept, trying to find a perfect combination of the many variables that define a particular instance. With the availability of interactive computer graphics tools, we now have the possibility to do much of this exploration and fine-tuning with virtual models, if we succeed in capturing our conceptual idea in the form of a computer program with an appropriate set of adjustable control parameters. Such virtual evaluation may be just a convenience when designing table-top sculptures; it becomes a crucial tool in the design of architectural sculpture.

In this paper we discuss our efforts to capture a variety of ribbed sculptures by Charles Perry [4] in this manner. By extracting an implicit framework that underlies most of his ribbed sculptures, we define a broad approach that enables a wide variety of new ribbed sculptures and mathematical visualization models. We will start our discussion with an analysis of Solstice, because it has a particularly compelling underlying representation that lends itself to an elegant parametrization.

As one walks around Perry's Solstice sculpture, one is amazed at the richness of diverse views that present themselves from different viewing directions (Figure 1). From some angles the sculpture looks like intertwined organic forms; other views inspire an association of a roller coaster on steroids. But from a few privileged vantage points, a wonderful symmetry is revealed, and the pattern becomes surprisingly regular. At this point an inquisitive mind just wants to know what is going on and whether there might be a simple generative principle that lies at the root of this elegant masterpiece. In the case of Solstice, this analytic task was made easy, since Charles Perry was quite forthcoming with explanations of how he planned this sculpture and how he went about constructing it. In a personal communication he wrote:

"The perimeter of Solstice is created by placing an equilateral triangle on a ring, so the centroid of the triangle connects with the ring. The triangle is rotated by two-thirds twists as it rotates around the ring. The figure produced by the three vertices of the triangle is a two-thirds twist torus Möbius.

Intuition told me of the right diameter of the tube for the edge of the torus. I made a 12 inch model and worked from that somehow. I found that there are four equal quarters going around the torus. I then made a full-scale mockup of 1/4th of the edge. It looked like a section of a roller coaster in my clean new studio. It was in three dimensions. I took this template to a tube-bender; they had a skilled old man who could bend the tube in compound curves to match the template. This was done in sections. In the studio I matched and welded these pieces. I had to cut off the excess ends. Now I had four equal tubes, probably about fifteen feet long. I then determined where the cross tubes would be closest to each other. This part was done by referencing the 1 -foot model.

Now, these holes for the cross tubes had to be a variable distance from each other and had to rotate around the edge tube as they progressed. Masking tape, Magic Marker and a center punch for each hole was the method. Certainly I had to measure the total length of each quarter edge and divide this into the number of holes. All the cross tubes are equal. There are more than 600 cross tubes, and thus more than 1200 roto-broached holes, each at a different angle. The four equal edge pieces and the 600 tubes were shipped separately to Tampa. -- I don't even know how I registered the four edge pieces when it was assembled in Tampa." 
Perry's description and images of Solstice from different angles (Figure 1) allow an easy construction of a generating paradigm that can be captured in a computer program. Some of the crucial parameters in this generative program can then be made into variables, and by setting these variables to new (somewhat constrained) values, novel sculpture designs of "the same kind" can be generated. Before any of these modified designs are sent to the machine shop, one has to make an artistic judgment, whether the new forms have enough aesthetic merit to warrant an actual construction. If the answer is affirmative, then many more details will have to be worked out about how exactly to bend the many ribs into their specified shape, and how to connect them to the supporting tubular rails. Considerable engineering effort goes into working out those details.

In this paper we are mainly concerned with the primary design aspect of variations of Solstice and of other ribbed sculptures by Charles Perry [4]. We have generalized this paradigm and captured it in several small computer programs that allow us to design a wide variety of such sculptures. Our new designs are presented in virtual form by means of computer graphics renderings. Most of the construction details and engineering issues are ignored at this stage. As the reader will see, even the geometrical design phase offers several intriguing puzzles and programming challenges.

\section{Geometrical Emulation of Solstice}

Based on the information obtained directly from Charles Perry, we know that the thicker tubular "guide rail" lies on the surface of a torus. In the case of Solstice, it forms a $(3,2)$ torus knot, i.e., the guide rail runs three times around the big loop of the torus and passes twice through its tunnel before it closes again onto itself (Figure 2a). The thinner "ribs" attached to this guide rail are not simply the edges of a rotating equilateral triangle; straight edges would look rather stiff. The ribs are planar curves, three of which form an approximate "hyperbolic" triangle composed of three inward-bending concave circular arcs. Furthermore, the ribs do not form closed, threesided, planar loops, but rather form a spiral staircase. The two ribs that would end in the same triangle vertex have been offset along the guide rail by half the distance between subsequent triangles, so that the ribs seem to land on the guide rails individually, with apparently uniform spacing (Figure 2b). Of course, on the inside of the torus the spacing is much denser than on the outside, since the truly relevant spacing parameter is the "equatorial" angle around the torus. Strictly speaking, this forces the geometry of the many ribs to vary ever so slightly. However, the geometrical deviations are small, and they are within the tolerances to which the actual tubular ribs can be

bent. Thanks to their curvature, the ribs can thus readily be fit into the toroidal guide rail at assembly time.

To capture this constructive paradigm, we wrote a program module to generate a guide rail in the form of a sweep surface along an arbitrary $(p, q)$ torus knot. The parametric representation of this sweep line, lying on a torus surface with big radius $R$ and small radius $r$, is:

$$
\begin{aligned}
& x=\left(R+r \cos \left(\frac{q \phi}{p}\right)\right) \cos \phi \\
& y=\left(R+r \cos \left(\frac{q \phi}{p}\right)\right) \sin \phi \\
& z=r \sin \left(\frac{q \phi}{p}\right)
\end{aligned}
$$

where $0<\Phi<2 p \pi$.

This loose frame is then populated with a parameterized set of ribs, which themselves are circular arcs. More explicitly, the guide rail is specified by the integer constants $p$ and $q$ of the torus knot, by the radii, $R$ and $\mathrm{r}$, of the major and minor circles that define the torus on which this knot is embedded, and by the diameter of the guide rail tube itself. 
Next we specify the total number of ribs and the offset of the two rib endpoints from an exact cross-sectional plane (cutting a minor circle from the tube of the torus). This offset is measured as an angle along the circular sweep path that defines the major loop of the torus. An offset of zero keeps the ribs entirely in the cross-sectional plane, and an offset of 1 degree would move one of the endpoints of the rib forward by that amount in the major sweep direction. Actually, in our programs this offset is typically defined as a fraction of the total sweep angle of the torus knot guide rail. This makes it easier to interleave properly the rib endings on the guide rail. For a total number of 500 ribs an offset of $0.1 \%$ would evenly stagger all rib endings with a minimal amount of helical twist.

The individual ribs themselves are circular arcs between their two end points on the guide rail. The amount of arching of each rib can be specified as the turning angle that this circular arch segment is bending through. Alternatively, the amount of bulging can be characterized by the maximal distance of the arc from the chord connecting the two rib endpoints. Different versions of our programs have used different approaches. In either case, the amount of bulging is normalized so that a unit of "1" leads to ribs that would hug the torus surface (at least for small values of the offset parameter); this normalization varies with the variable $p$. A "bulge" of zero always results in a straight rib, and a negative bulge value indicates that the arc is curving in the inward direction (Figure 2c).
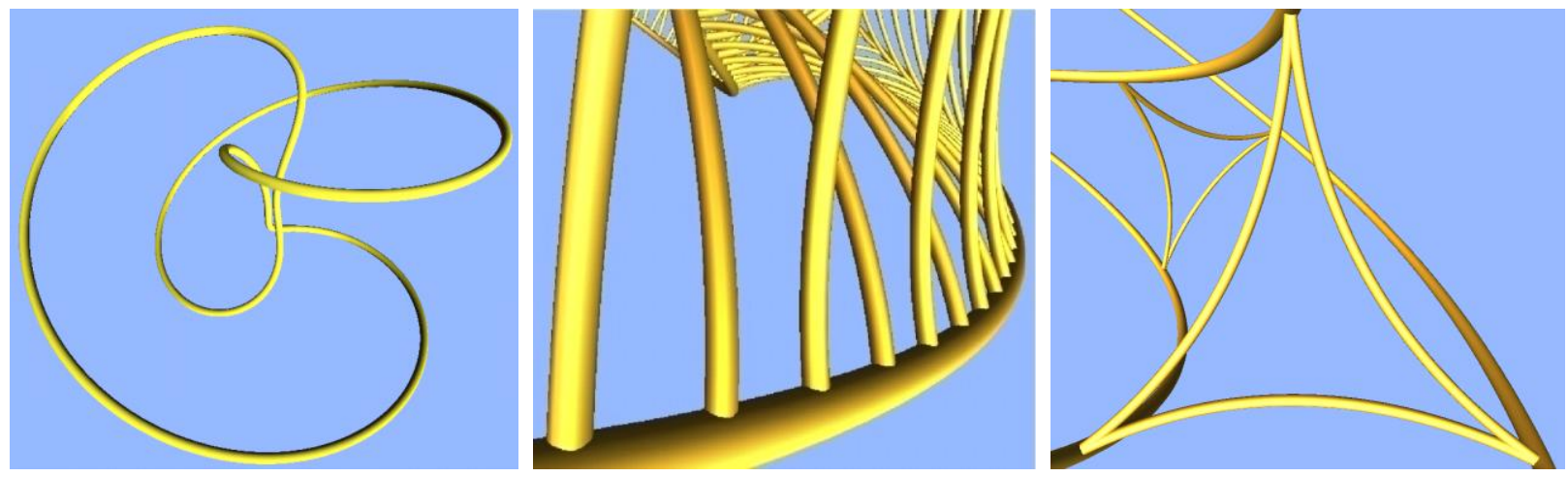

Figure 2. Graphical illustration of the key design parameters in the Solstice program: (a) the torus knot formed by the guide rail, (b) a rib-end offset resulting in "evenly" spaced ribs, (c) a negative bulge of the individual ribs.

By tuning all the above mentioned parameters carefully, a rather faithful emulation of Perry's Solstice sculpture is obtained. In the emulation shown in Figure 3 the program parameters listed in Table 1 were used. (As far as the number of ribs is concerned, we guess that Perry's memory was off by a factor of 2.)

Table 1. Program parameters used in the emulation of Perry's Solstice sculpture

\begin{tabular}{|l|l|}
\hline$p$ & 3 \\
\hline$q$ & 2 \\
\hline$R$ & 6.5 \\
\hline$r$ & 6.0 \\
\hline Guide-rail diameter & 0.15 \\
\hline Rib diameter & 0.1 \\
\hline Number of ribs & 300 \\
\hline Rib offset & $1.8^{\circ}$ \\
\hline Rib bulge & -0.5 \\
\hline
\end{tabular}



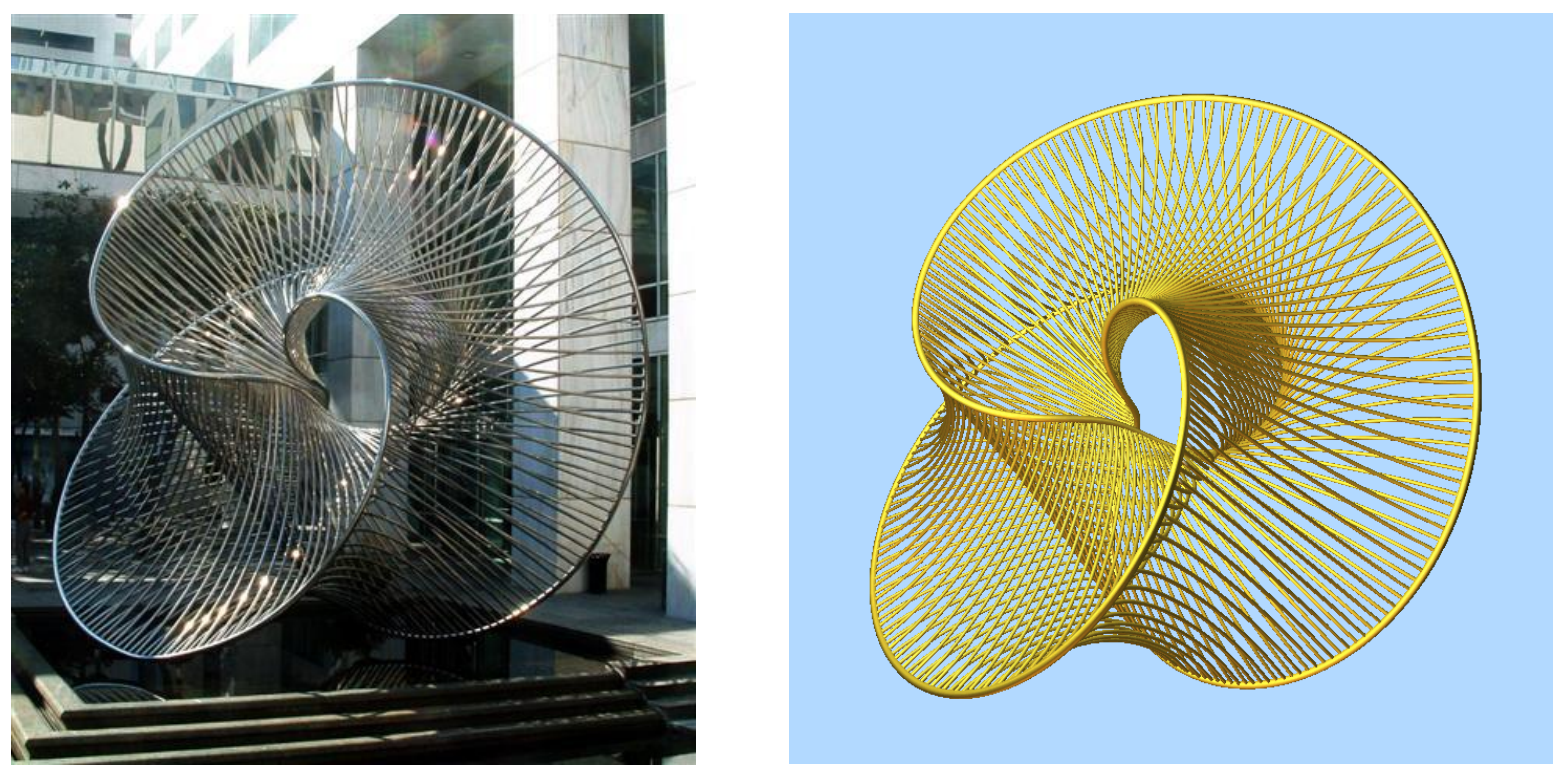

Figure 3. Emulation of Solstice: (a) Perry's sculpture in Tampa [Photo copyright C. H. Séquin, 2007], (b) computer emulation shown from the same angle.

\section{Solstice Variations}

With the basic generating paradigm captured in our program, it is now easy to make variations of this sculpture. In a first example we reduce the amount of twisting in the overall toroid to obtain a shape more closely related to Helaman Ferguson's bronze sculpture called Umbilic Torus NC [1], in which the total twist of the triangular cross section is only $120^{\circ}$ (Figure 4a). This can be readily achieved by changing the parameter $q$ to 1 , yielding a guide rail in the form a $(3,1)$ torus knot - which is actually not knotted at all.
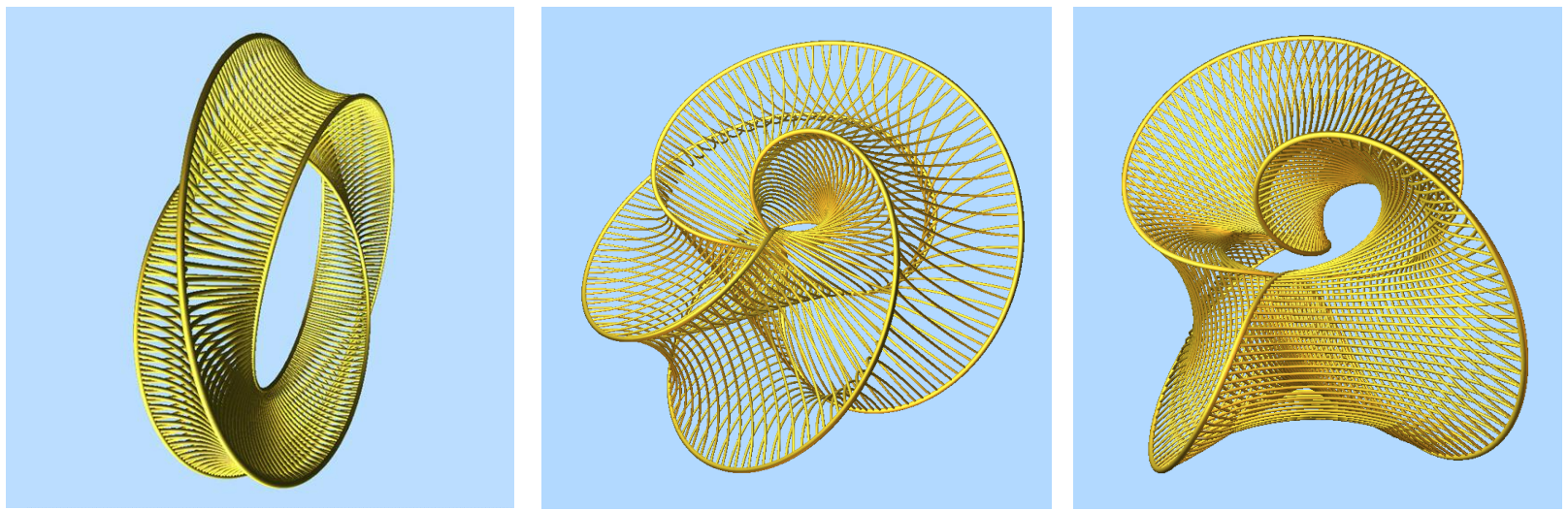

Figure 4. Solstice variations: (a) emulating the $(3,1)$ torus knot of Helaman Ferguson; (b) $(4,3)$ torus knot variation; (c) $(2,3)$ torus knot variation.

Alternatively we may choose to increase the twist in the toroidal sweep structure. Avoiding the case $(3,3)$, where the guide rail would break up into 3 separate loops, the next connected candidate is the $(3,4)$ torus knot. This however looks too twisty for our taste; instead we explore the case of the $(4,3)$ torus knot. This results in a quadrilateral cross section that makes a 3/4 turn while traveling once around the toroidal sweep (Figure 4b). This configuration certainly has the potential for another large-scale ribbed sculpture. 
Another experiment is to simply switch the values of $p$ and $q$ of the original Solstice, thereby generating a $(2,3)$ torus knot, while leaving the rib specifications unchanged. Even though topologically the structure of the knot has not been changed by this switch, the result now looks quite different (Figure 4c). There are two main reasons: First, the geometry of the guide rail now has a totally different structure, even though it describes the same mathematical knot; second, the behavior of the ribs has changed dramatically. There are now only two passes of the guide rail through every minor circle of the torus, and this is not sufficient to form a rib triangle. Also, retaining the parameterized rib endpoint values of the original Solstice leads to an effective angular offset of about $120^{\circ}$. This happens because the rib endpoints are specified by a parameter that relates to the whole length of the guide rail. While in the original Solstice one third of the length of the rail completed one sweep around the torus, in this new variant with only two major loops in the guide rail, it brings the second rib endpoint only about $2 / 3$ around the torus. Thus the new configuration of the ribs yields the looks of a puffed up cushion and adds more "volume" to the appearance of the sculpture.

\section{Other Ribbed Sculptures by Charles Perry}

Charles O. Perry has created several other Ribbed Sculptures [4] that can be modeled with this paradigm. His sculptures inspired us to extend and generalize the generating paradigm of populating one or more guide rails with a set of closely spaced ribs. First we made a few modified versions of the Solstice emulation program to capture the geometries of some other Perry sculptures. Keeping the programs separate, kept them light-weight and easyto-modify, and is preferably to a monolithic heavy-weight program in this early phase of exploration.

Ribbed Mace (1998), located in Falls Church, VA (Figure 5a), is probably the simplest of Perry's ribbed structures, yet it is definitely eye-catching. It uses two separate semi-circular guide rails in planes that stand at right angles to each other. Its 49 straight ribs form an elegant ruled surface that connects the two guide rails. In our first emulation we have slightly increased the number of ribs and placed them evenly spaced onto the guide rails (Figure 5b). Figure 5c shows a variation with curved ribs, which seems to give the sculpture a more lightweight, wing-like look.
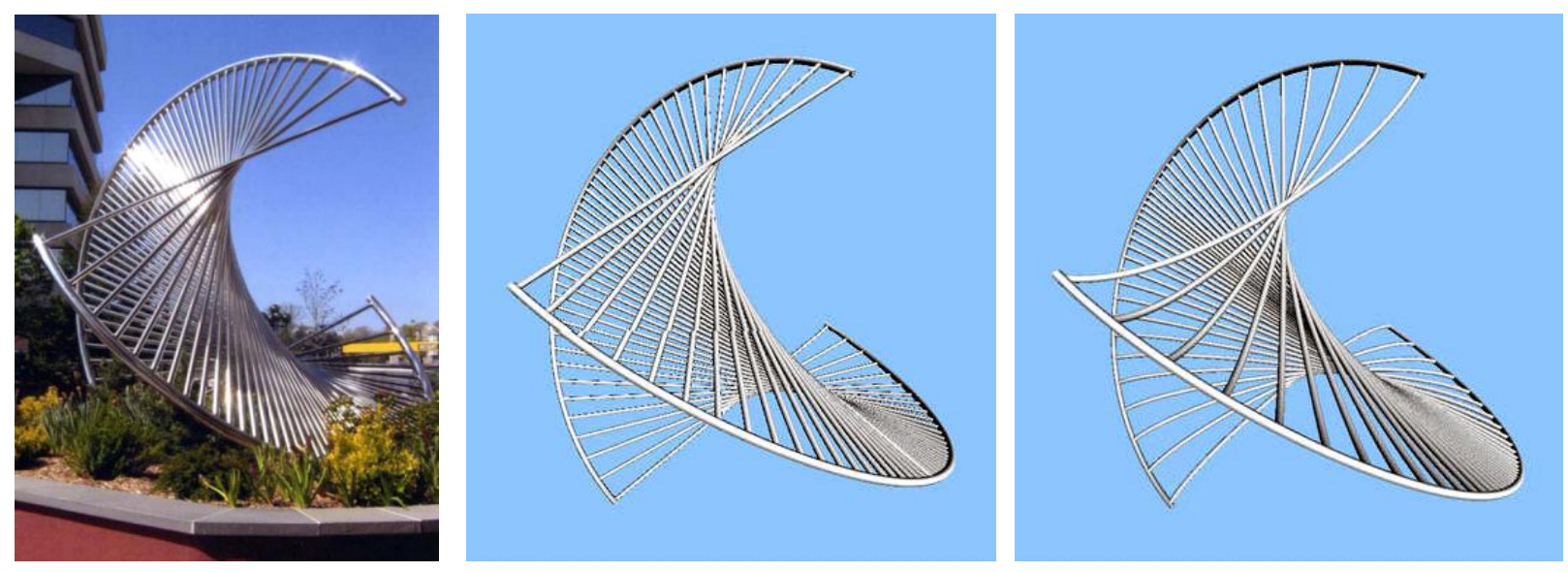

Figure 5. Ribbed Mace: (a) Perry's sculpture [Photo copyright C. Perry, 1998], (b) emulation (differing rib density), (c) variation with curved ribs. 
Harmony (1990), located in Hartford, CT (Figure 6a), also uses two separate semi-circular guide rails. However, it comprises four distinct ribbed surfaces, each composed of 18 ribs of varying curvature. Figure $6 \mathrm{~b}$ shows our emulation of this sculpture, but with 21 ribs in each of the four sets.
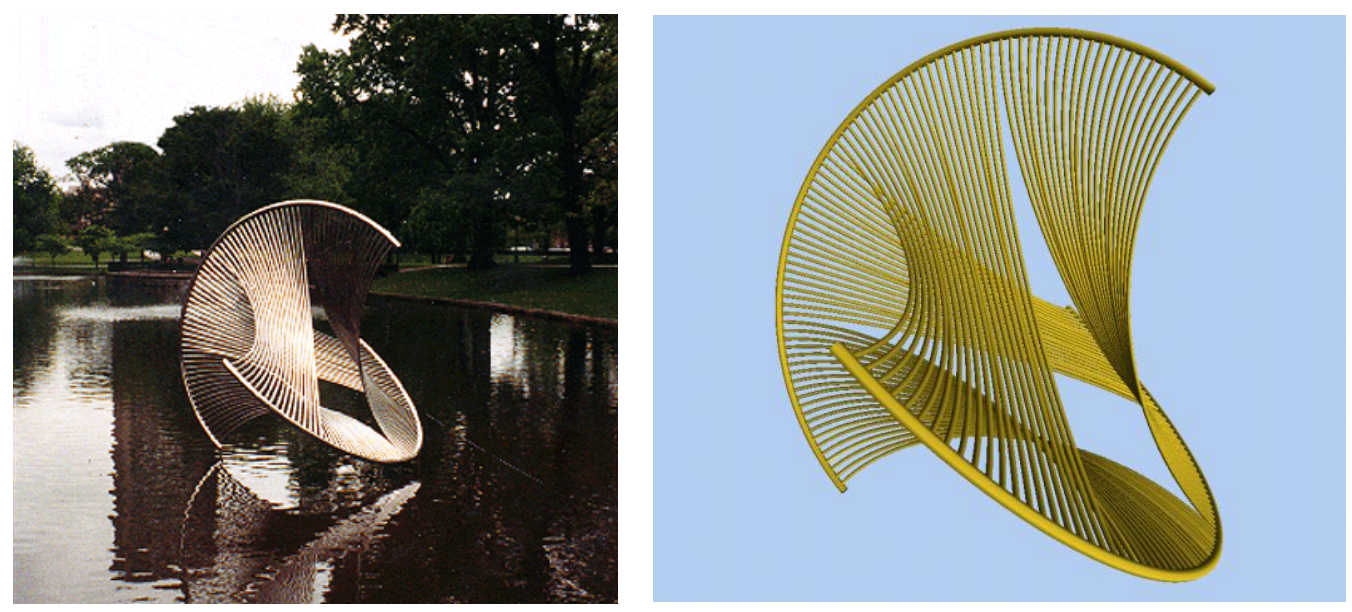

Figure 6. Harmony: (a) Perry's sculpture [Photo @) Perry, 1990], (b) an emulation with higher rib density.

Early Mace (1971), located in Atlanta, GA (Figure 7a), uses ribs in the shape of circular arcs connecting two separate guide rails. In this case the rails are two pairs of (almost) great semicircles on an invisible sphere, held together by two small semicircles at both ends; this gives the mace shape some thickness. The ribs form inwardsbending quarter arcs. Figure $7 \mathrm{~b}$ shows an emulation of this sculpture, while Figures $7 \mathrm{c}$ and $7 \mathrm{~d}$ demonstrate what happens when the ribs are first straightened and finally bent outward to follow the surface of the sphere, respectively.
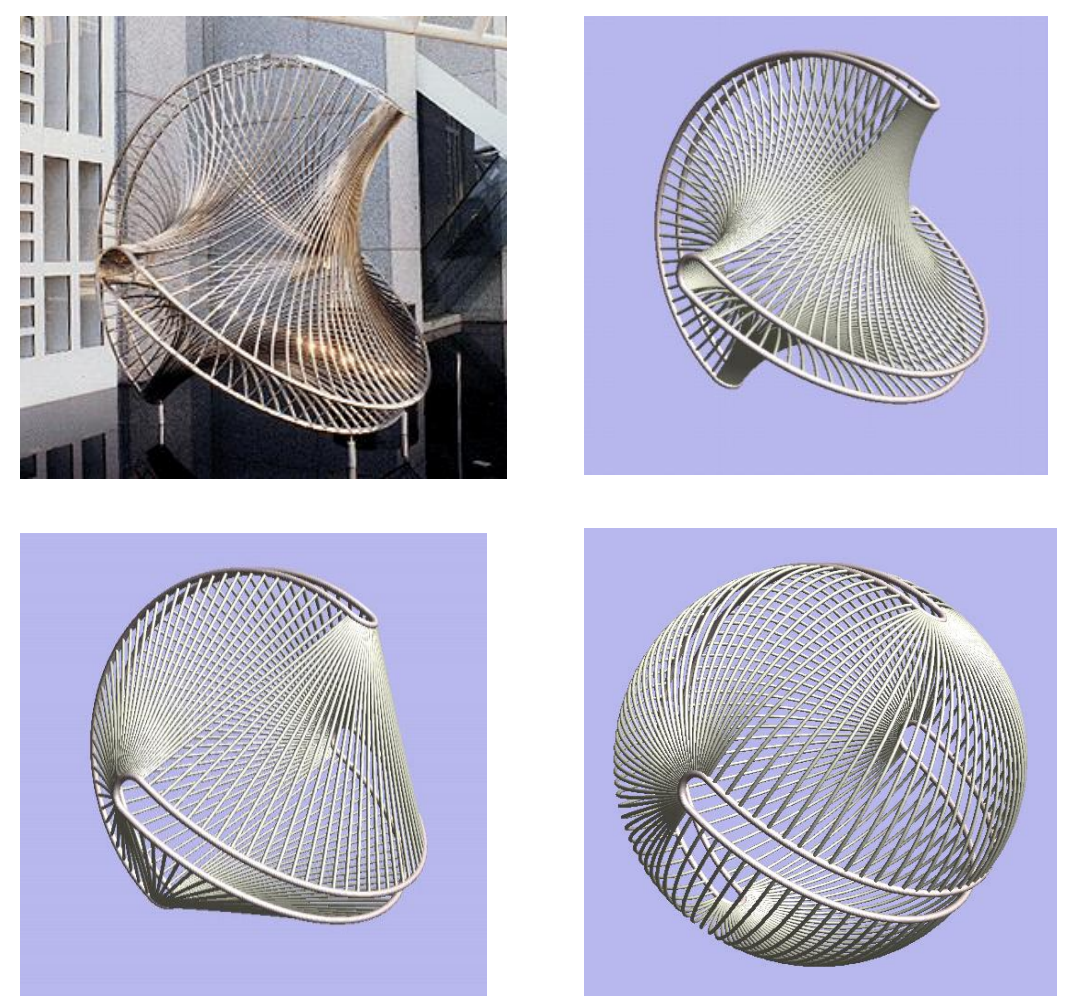

Figure 7. Early Mace: (a) Perry sculpture [Photo @ Perry, 1971], (b) emulation, (c)(d) rib variations. 
Eclipse (1973), located in San Francisco, CA (Fig. 8a), is a much more complicated ribbed structure. It is a construction with dodecahedral symmetry, where each face of the famous Platonic solid with 12 pentagons is replaced with a tapered and twisted "nest" of curved pentagonal rings. Again, the geometry of these rings, as well as their scale and relative offset from one ring to the next one, can easily be captured in parameterized form and exposed to experimentation. In a graduate course on "Computer-Aided Design and Rapid Prototyping" at U.C. Berkeley [8] some students played with this design and created new versions of the "nest" geometry (Fig. 8b).
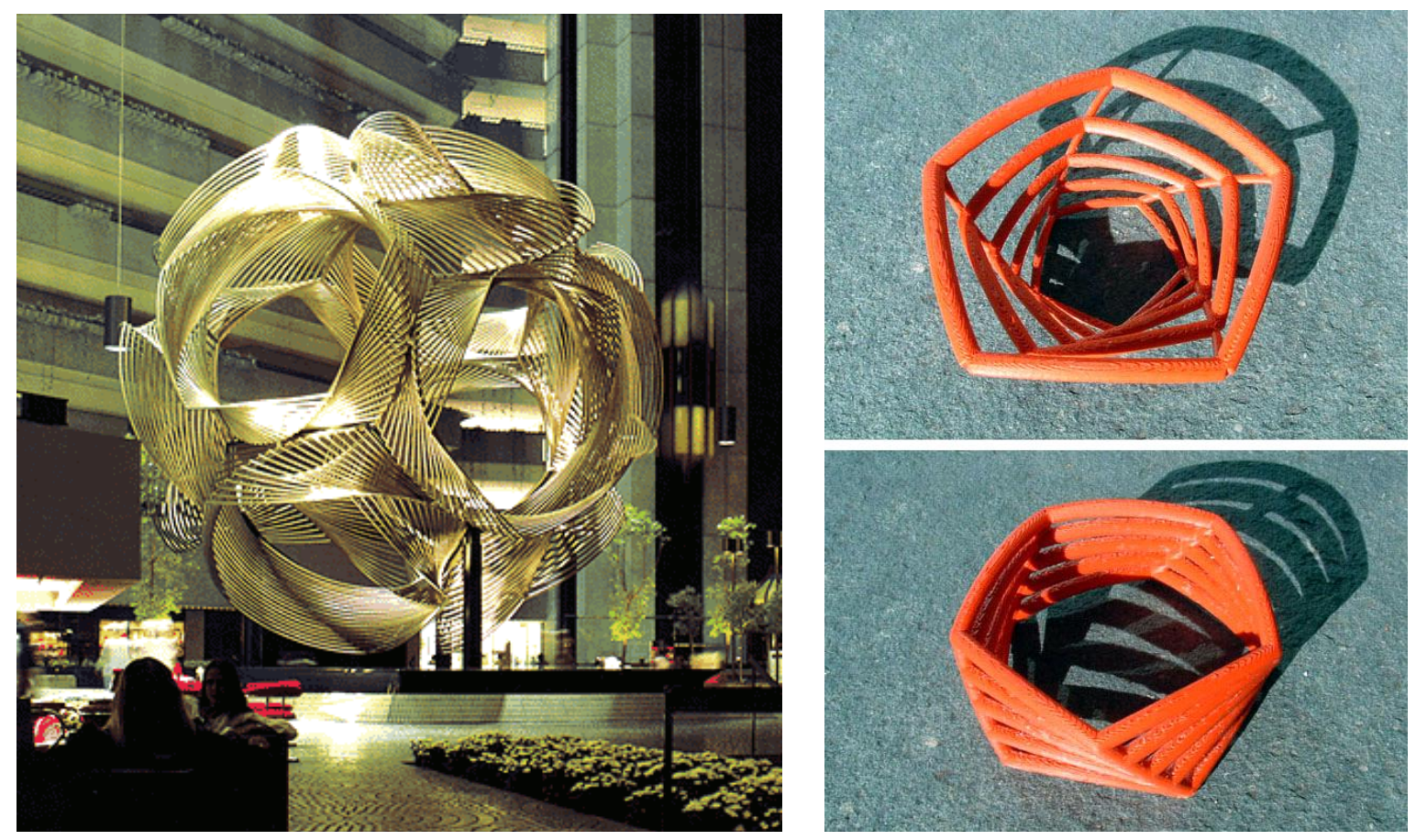

Figure 8. Eclipse: (a) Perry's sculpture [Photo copyright C. Perry, 1973], (b) and (c) modified "nest" modules.

We have also captured the core features of Perry's Eclipse in an emulation program using ribbed surfaces. We allow the user to vary the number of ribs, the degree of twist in each "nest", and the recursive scaling of the extruded pentagons that forms each nest. Figure 9 demonstrates the variety of forms that one can create with these simple parameters. Figure 9a shows a ribbed dodecahedron with cylindrical nests with a moderate twist. Figure $9 \mathrm{~b}$ sows a view from the inside of such a structure. Figure $9 \mathrm{c}$ is another variant with highly twisted conical nests, which scale down the extruded pentagons as they move outwards and thereby form twelve pointy spikes.
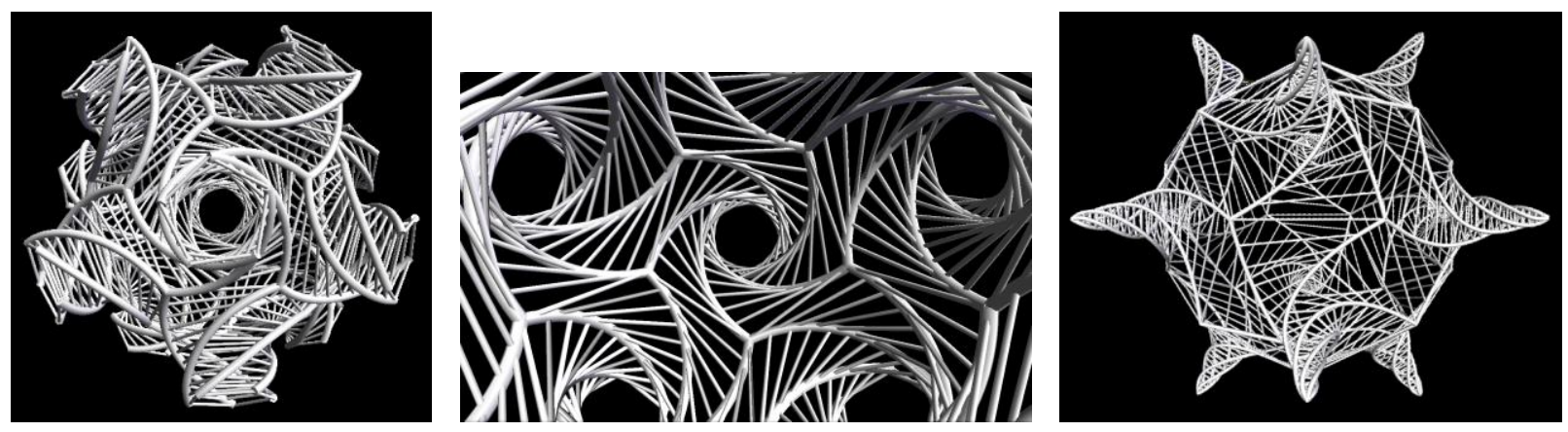

Figure 9. Variations on Perry's Eclipse: (a) 12 cylindrical nests around a dodecahedron, (b) inside view of such a structure, (c) highly twisted, pointy nests. 


\section{Generalized Ribbed Surfaces}

In order to create a general ribbed surface [2],[3], we need to define either one or two parameterized guide rail curves and the rail cross-sections that will be swept along the rail curve(s). For the ribs we have to specify the total number of ribs, their cross-sectional shape, and two parameter intervals for the locations of their endpoints beginning on guide rail $\mathrm{Gb}$ and ending on guide rail Ge. For example, if there are two separate guide rails, $\mathrm{Gb}$ and $\mathrm{Ge}$, for the rib endpoints, the beginnings might be spread over the interval $[0,0.5]$ on rail $\mathrm{Gb}$, while the endpoints span the interval: $[0,1.0]$ on Ge (Figure 10a).

We must also provide an application-dependent set of geometric functions that define the sweep curves for the individual ribs spaced out along the rails. We have employed several alternative coordinate systems associated with the end points of the ribs, which allow us to specify the rib shapes in the most convenient manner for a particular sculpture family [2]. For instance, when the ribs are represented as cubic Hermite curves, the ribs are specified by their two endpoint positions and their two end tangent vectors. The endpoints are simply points along the guide rail curves, evaluated at the proper parameter values of the guide-rail sweep for that rib. The end tangents might point in any direction, and different coordinate systems have different advantages for specifying these tangent vectors, as will be discussed below. The orientation angles of the end tangents in these rib-local coordinate systems may vary as a function of the sweep parameter $s$. In the simplest case, the rib end-condition parameters are specified for the start and for the end of the guide-rail sweep and are linearly interpolated for all ribs in between. More complicated functions could easily be introduced.

The most general approach simply uses the Frenet frame of the rail curve as the coordinate system to define a rib end tangent vector. The rib endpoints are placed at the origin of this Frenet frame, and the rib endpoint tangents $v$ are defined in terms of the rail curve tangent $(t)$, normal $(n)$, and binormal $(b)$ (Figure 10b).

Often we are more concerned with the overall shape of each rib itself, rather than the way it lands on the guide-rail. In this case we specify the geometry of the rib endings in a coordinate system that is more intimately tied to the bulk of the rib. The chord that connects the two end points of the rib sliding along the rails forms the dominant axis of such a coordinate system. A plane that passes through this dominant axis can be specified by a single angle parameter $\rho$. The plane defining the zero angle depends on the shape of the guide rails; as a default we may try to keep it as close as possible to perpendicular to the rails at both rib endpoints.

In many cases, it is also desirable to keep the ribs planar and symmetric. In this case all that needs to be specified is the orientation angle $\rho$ of the rib plane around the dominant coordinate axis and the tangent angles $\tau$ that the rib ends form with the chord. Alternatively, the amount of bending of the rib in the given plane can be specified as an offset distance $d$ of the rib mid-point from the chord mid-point (Figure 10c).
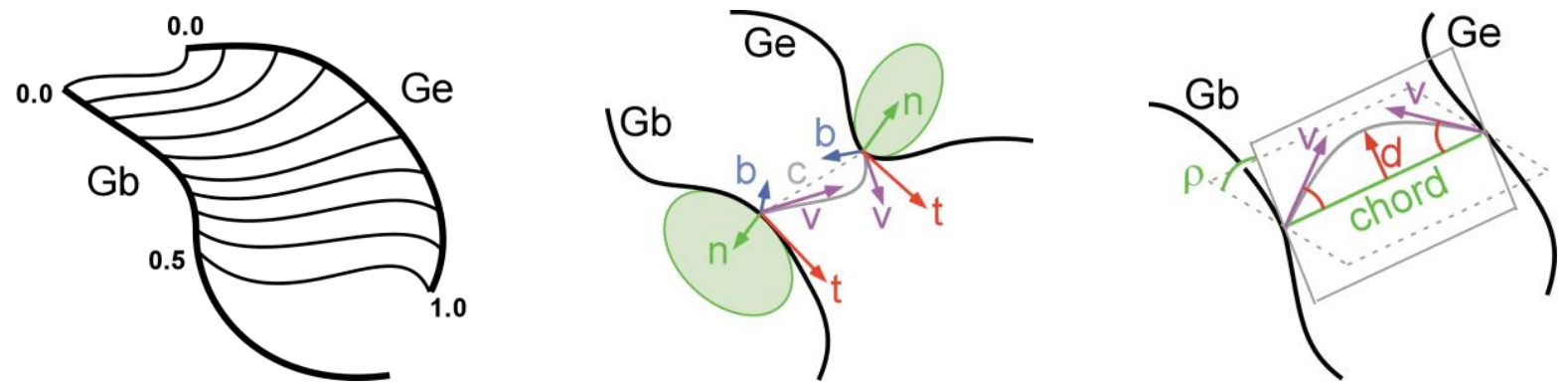

Figure 10. Different rib specification schemes: (a) basic concept: sequence of ribs spanning two rail, (b) rib specification based on Frenet frames at end points, (c) planar ribs as a modification of the chord between end points.

The most suitable parameterization of the rib end conditions is application-specific and may reflect both aesthetic and pragmatic requirements. For example, if planar ribs approximating elliptical arcs are the preferred solution, the second coordinate system described above might be used (Figure 10c), perhaps with a further abstraction to 
specify the rib shape in terms of elliptic eccentricity. Other ways of defining the geometry of the individual ribs may use a blend of the two systems described here [2].

\section{Ribbed Surfaces and Mathematical Visualization}

"String Art" models of mathematical surfaces depicting hyperbolic paraboloids and other ruled surfaces (Figure 11a) are found in many science museums. Because of their "transparency" such ribbed surfaces are particularly useful when depicting complex, possibly self-intersecting, geometrical objects. Figures $11 \mathrm{~b}$ and $\mathrm{c}$ show ribbed models of a hemi-cube and of a hemi-dodecahedron. These are non-orientable, generalized polyhedra (or 2D cellcomplexes) that can be obtained by taking surface elements of the cube or of the dodecahedron and identifying antipodal points, edges, and faces. Thus the 3-dimensional visualization of the hemi-cube has just three bilinearly warped "squares" (Figure 11b), and the hemi-dodecahedron is composed of six warped pentagons (differently colored in Figure 11c), forming a tetrahedral structure with six angled edges. The latter is the basic building block of the 4-dimensional 57-Cell [9]. Slight modifications of one of the programs described above can readily be used to generate such mathematical visualization models and to build them on a rapid-prototyping machine (Figure $11 b)$.
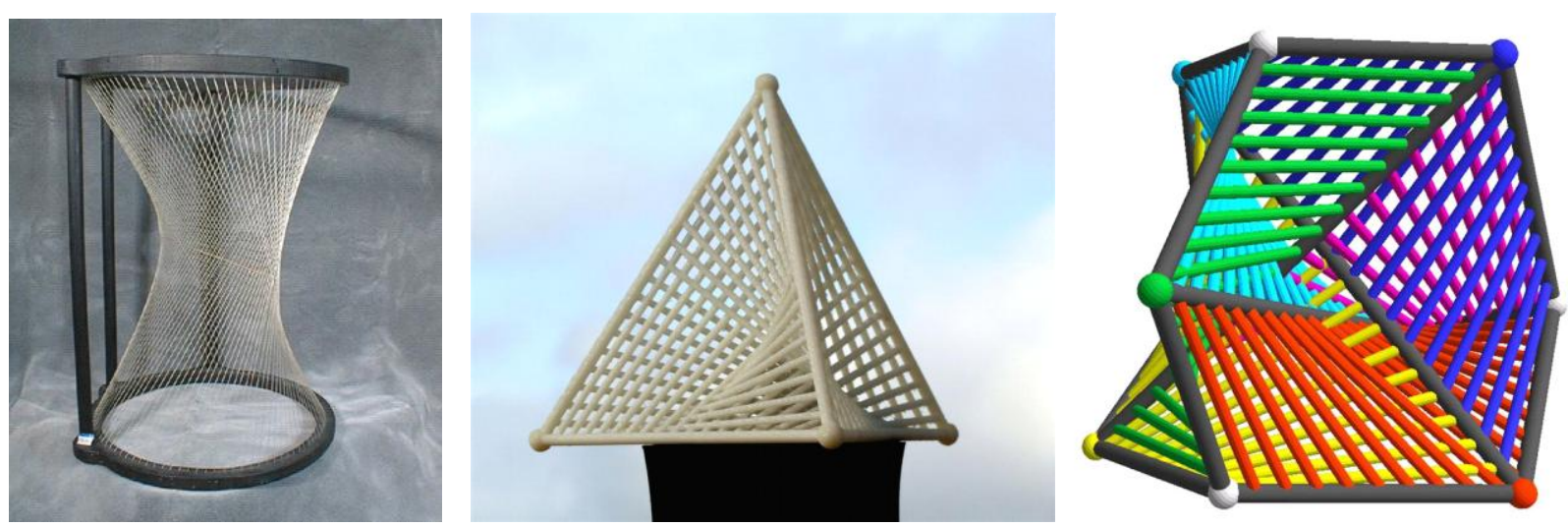

Figure 11. Mathematical models: (a) Hyperboloid, (b) hemi-cube, (c) hemi-dodecahedron.

\section{Designing Virtual Ribbed Sculptures}

Using the generalized paradigm of ribbed surfaces, we have created some parameterized sculpture models of our own design. First, we present the design of one of these sculptures step-by-step to show how one may use the discussed broad concept to arrive at a pleasing sculptural form with a level of complexity that makes an uninitiated viewer wonder how this structure may have been conceived.

\section{Figure-8 Knot}

Knotted curves are ideally suited as guide rails. Thus we begin by defining a guide rail in the shape of a symmetrical presentation of the Figure- 8 knot (Figure 12a). Since we are working with a closed curve, rib placement is reduced to specifying a single "offset" parameter, that specifies how far apart (in a fractional sense) are the two endpoints of each rib. Starting from the already interesting topology of the selected knot, we now introduce additional structured complexity by adding one or more ribbed surfaces.

Inserting ribs that span a significant fraction of the knotted rail curve can produce rather confusing results. Thus, to begin with, we use a rather sparse set of thin ribs, so that the structure of the rail curve remains dominant. Figure 12a shows what happens when we introduce a few skinny ribs that span exactly $50 \%$ of the Figure- 8 rail curve. Because of the inherent symmetry of the chosen knot representation, all ribs remain parallel to the ground plane and thus result in an easily comprehensible but fairly dull and unattractive "ladder." 
As we change the rib offset to about $25 \%$ of the total rail length, a more interesting pattern emerges, creating a nicely structured surface with ribs that point in many different directions (Figure 12b). Figure 12c shows this same structure from the top.
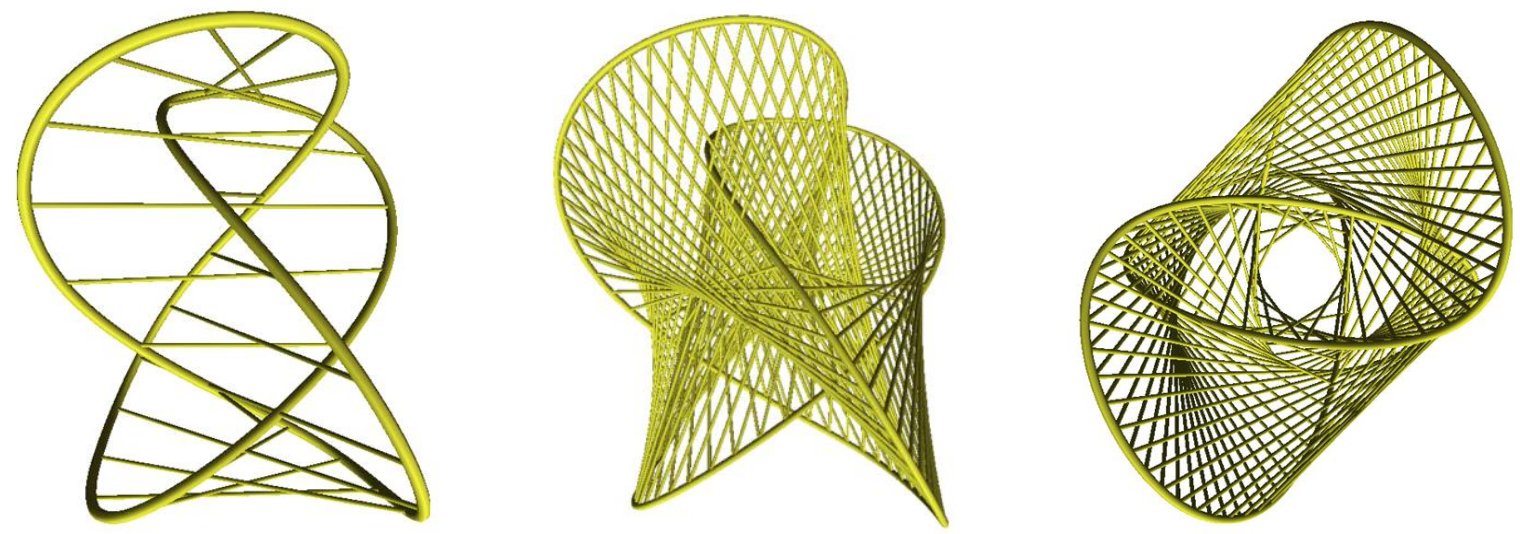

Figure 12. Figure-8 Knot: (a) Guide rail with just a few ribs with an offset of 50\%, (b) and (c) the resulting structure with many more ribs with an offset of $25 \%$, seen from the side and from the top.

Up to this stage of the design process, the intervals between the rib endpoints along the guide rail have been left to chance, and they may actually collide (Figure 13a, top). We now fine-tune these rib endings, aiming for evenly interleaved ribs as in Perry's Solstice (Figure 13a, bottom). To obtain a precise staggering, we want our offset to be a multiple of $(50 / n) \%$, where $n$ is the number of ribs. With a few extra lines of code in the sculpture design program, this special constraint can be maintained automatically.
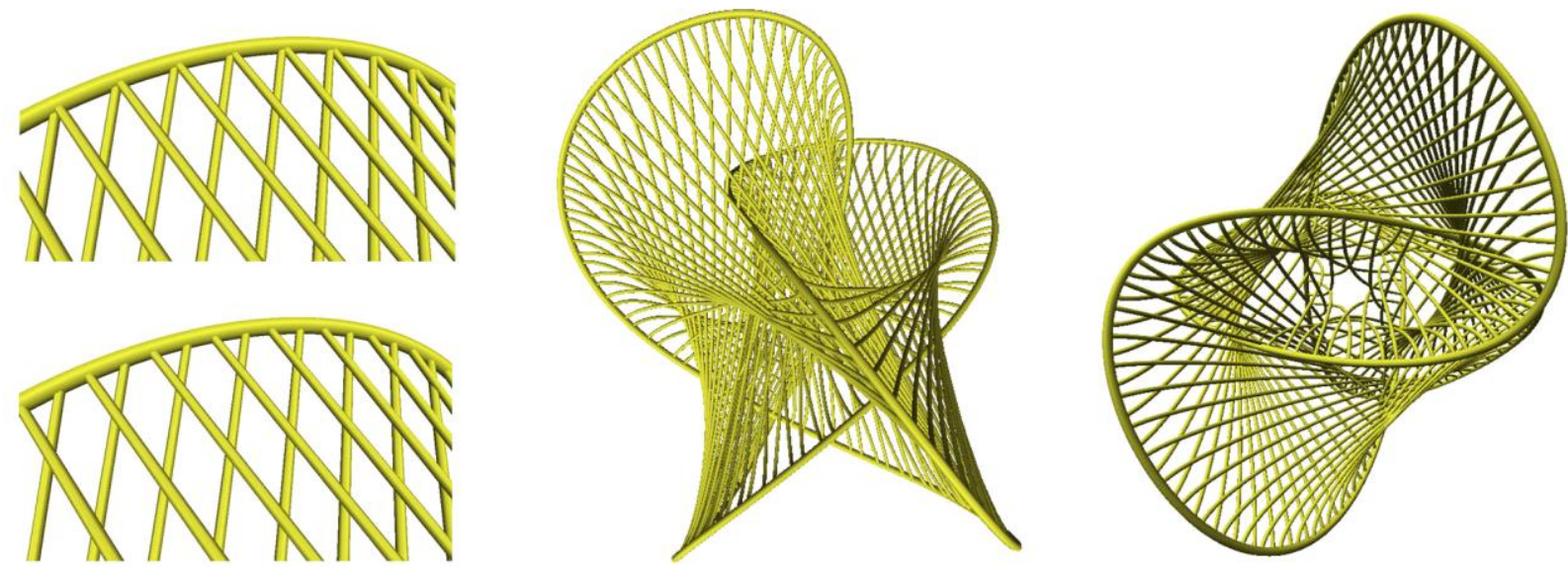

Figure 13. Adjusting the rib endpoint spacing: (a) coinciding versus evenly spaced endpoints, (b) and (c) refined Figure-8 Knot sculpture with curved ribs, seen from the side and from the top.

So far in this example we have used only straight ribs. Now that we have achieved a satisfactory configuration of the rib endpoints, we can start to fine-tune the rib shapes. In this example we use a cubic Hermite representation for the rib curves and manipulate the endpoint tangent vectors in the Frenet frame of the rail curve at each endpoint. For example, if the end tangent vectors of the ribs are kept aligned with the local normal vector of the rail curve, the ribs would take off from the apex of one of the "lobes" of the Figure- 8 knot in the direction towards the center of that lobe. A slider controls by how much this tangent vector deviates from the osculating plane of the guide curve. Our refined sculpture design is shown in Figures $13 \mathrm{~b}$ and c. 


\section{DNA Arch}

In a second experimental design we use hemi-elliptical ribs spanning double helix guide rails that are bent into a parabolic arch. The two guide rails forming the double helix twist around one another by a variable number of times, which can be set via a slider. The radius of the intertwined helices is specified separately at the end-points and at the apex of the arch and varies smoothly between these values. The double helix is curved to follow a parabolic path, which is specified with a few parameters that define the overall arch structure: its height and the width of its base. The rib end conditions are specified using a more general coordinate system based on the chord vector, the guide rail tangent vector, and their cross product (Section 5); the ribs take off in the direction of this cross product. These end conditions are specified at the two ends of the arch so that the ribs to take off in the upward direction, away from the ground plane. These end-condition values are then interpolated for each rib along the whole arch. Figure 14 shows two variations of such sculptures output by this program.
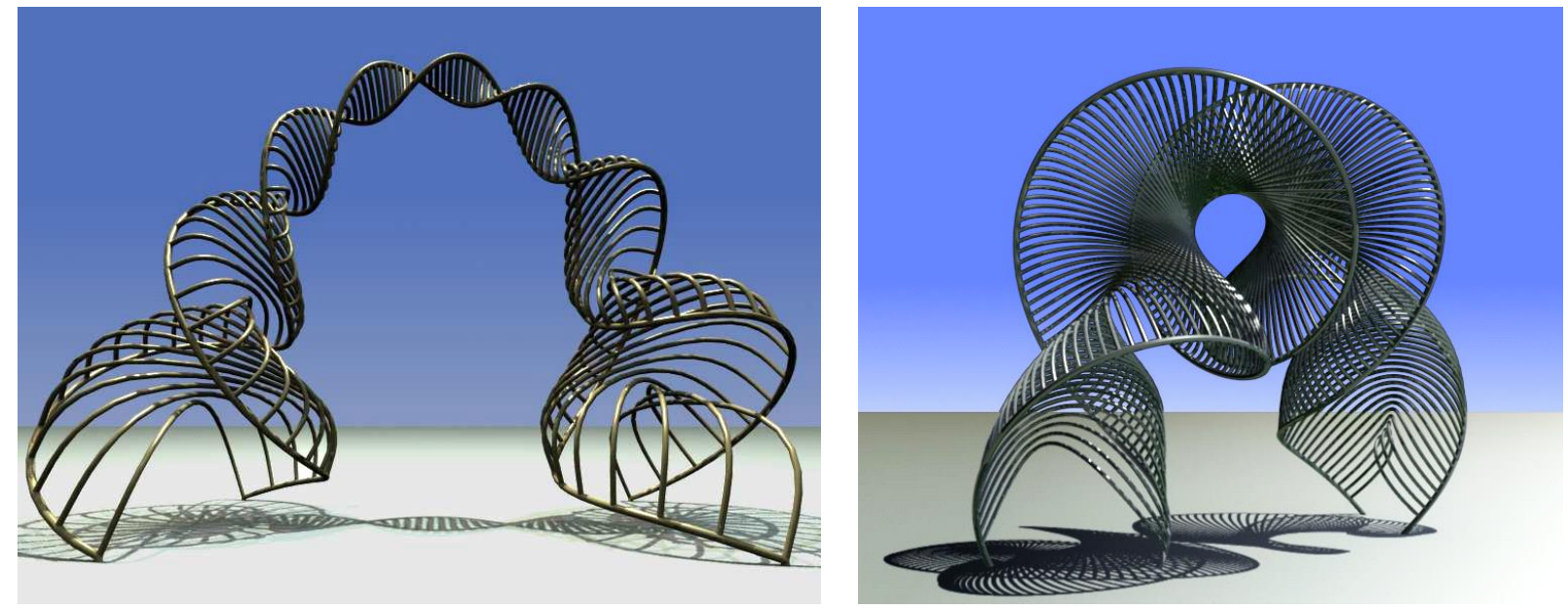

Figure 14. Two variations of the DNA-Arch sculpture.

\section{Rendering}

Finally, we briefly comment on the rendering of the images of our sculpture models presented in this paper. The geometry of our sculptures is defined in relatively small, special-purpose programs that have been written explicitly to capture a particular type of ribbed surface. Some of them were stand-alone C++ programs; others were special modules added to our SLIDE design environment [10]. None of these have any sophisticated rendering capabilities; they are just sufficient to make visible the emerging geometry during the interactive design phase. Once the geometry has been well defined, one would sometimes like to make a more realistic looking rendering of a proposed sculpture, ideally immersed in the environment for which a particular sculpture may have been designed.

For these higher-quality renderings we used the open-source RenderMan implementation Pixie [5]. Our programs support output in RenderMan's RIB format, and this output is then augmented with environment geometry and images. For example, in Figure 15, we used photographs taken on the UC Berkeley campus to produce reflection maps and a believable backdrop for a rendering of our Solstice emulation. 


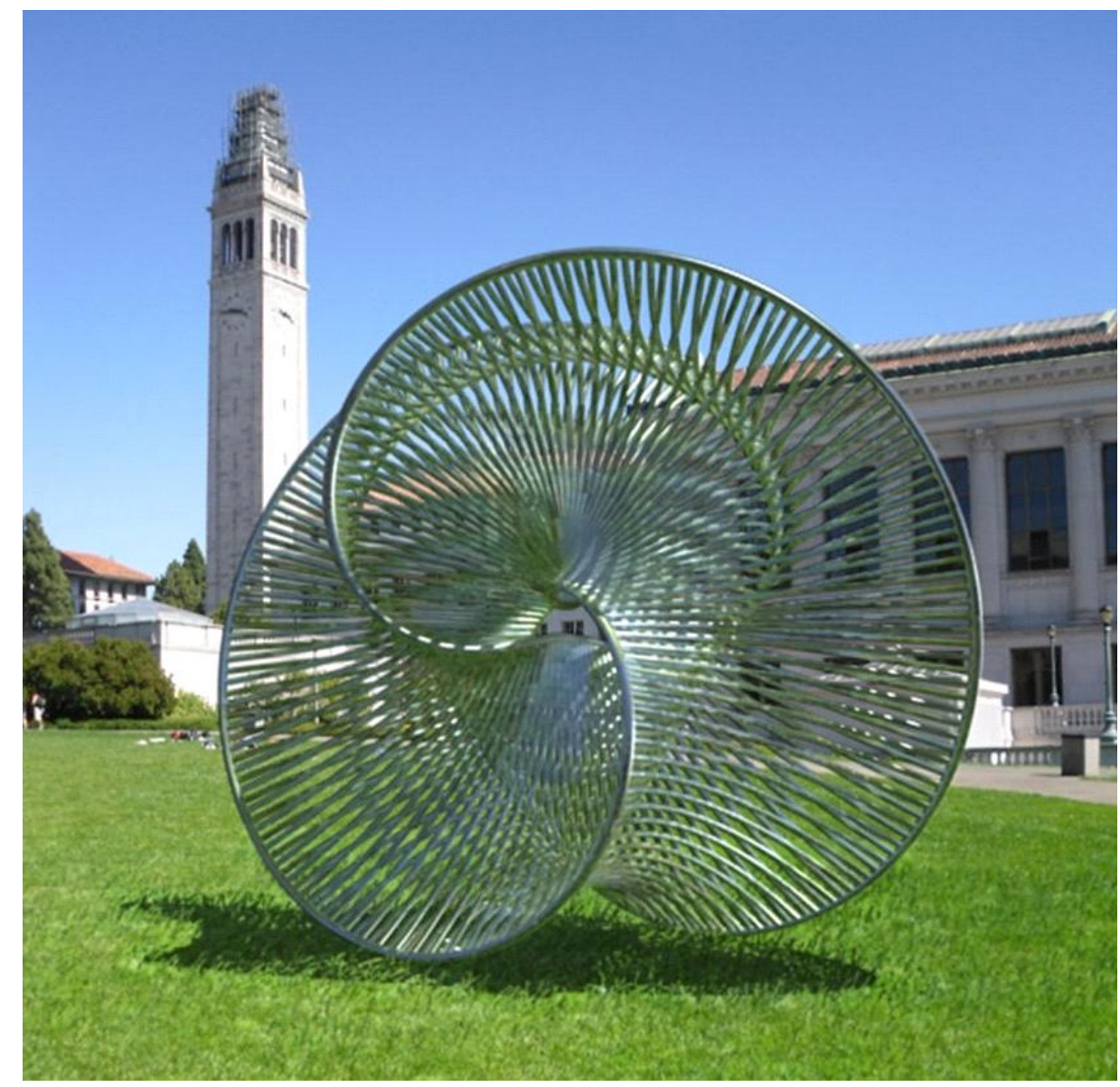

Figure 15. Virtual rendering of a sculpture immersed in the central glade of the Berkeley campus.

\section{Discussion and Conclusions}

With enough effort, any natural or man-made form can be modeled with computer graphics techniques as a oneof-a-kind effort. Our efforts described in this paper were focused on capturing a whole family of similar shapes with a single parameterized representation. The ensuing programs should not only reproduce the inspirational sculptures from which we started with reasonable accuracy, but should then open a rich playing field for further experimentation with the extracted paradigm. Ideally these programs provide a judicious user with a powerful tool with which additional aesthetically pleasing forms can be created, that still belong into the same shape family.

We believe we succeeded quite nicely in defining a few basic geometrical operators that jointly are able to faithfully reproduce some of Perry's ribbed sculptures, and which can generate other attractive sculptural forms. However, the existence of such a tool does not automatically turn every user into an accomplished artist - just like the emergence of photographic cameras did not prompt every user to turn out nothing but successful "lightpaintings." Once again we noticed, what had been observed previously with generator programs such as Sculpture Generator I [6] or with the SLIDE environment tailored to make a variety of Viae Globi sculptures [7]: Novice users typically are tempted to embrace too much complexity and produce what one anonymous reviewer aptly characterized in the following way: "If they begin with baroque they segue inexorably to rococo and oblivion." Indeed we have observed again this tendency among casual users to push some of the discrete variables of the program towards higher numbers, producing results that look more like a pipe-maze in a power plant than an inspiring sculpture.

In the work presented here this extraction and generalization has taken place at two distinct levels. First there is the analysis of Perry's Solstice as one member of a much larger family of "ribbed" $(p, q)$ torus knots, leading to 
the possibility of experimenting with different values for $p$ and $q$, but also altering the rib shapes and the offset of their endpoints along the torus-knot guide rail. Second there is the broader generalization of the notion of ribbed surfaces, composed of any set of slowly varying rib curves supported by one or more arbitrary guide rails. The combination of these two levels of abstraction leads to a fertile domain of intriguing geometrical shapes, which may make informative mathematical models as well as exciting monumental tubular sculptures.

\section{Acknowledgements}

We are indebted to Charles Perry for giving us permission to use the pictures of some of his ribbed sculptures and for letting us reveal the underlying logic of his Solstice sculpture by capturing its generating paradigm in a computer program, which then allowed us to create sibling designs to his masterpiece.

\section{References}

[1] H. Ferguson, Umbilic Torus NC.(1988). In Helaman Ferguson-Mathematics inStone and Bronze, Meridian Creative Group, Erie, PA, pp 6-7, (1994); see also: http://www.helasculpt.com/gallery/umbilictorusNC27inch/ (accessed April 10, 2010).

[2] J. F. Hamlin, Applications of Ribbed Surfaces. Master's Thesis, U.C. Berkeley (2009): http://www.eecs.berkeley.edu/Pubs/TechRpts/2009/EECS-2009-123.html (online library, accessed April 10, 2010).

[3] J. F. Hamlin and C. H. Séquin, Ribbed Surfaces for Art, Architecture, and Visualization. Computer-Aided Design \& Applications, Vol 6, No 6, pp 749-758, (2009).

[4] C. Perry, Ribbed Sculptures. (1966-1998): http://www.charlesperry.com/Ribbed.html (accessed April 10, 2010).

[5] Pixie rendering software: http://www.renderpixie.com/ (accessed April 10, 2010).

[6] C. H. Séquin, Interactive Generation of Scherk-Collins Sculpture. Symposium on Interactive 3D Graphics, Providence RI, pp 163-166; ACM, New York (1997).

[7] C. H. Séquin, Viae Globi - Pathways on a Sphere. Proc. Mathematics and Design Conference, Deakin University, Geelong, Australia, July 3-5, 2001, pp 366-374; see also: http://www.cs.berkeley.edu/ sequin/PAPERS/MD2001_ViaeGlobi.pdf (accessed April 10, 2010).

[8] C. H. Séquin, Rapid Prototyping of CAD Models. CS Graduate course 294-10, Fall 2003: http://www.cs.berkeley.edu/ sequin/CS294/ (accessed April 10, 2010).

[9] C. H. Séquin and J. F. Hamlin, The Regular 4-Dimensional 57-Cell. Presented at SIGGRAPH'07, Sketches and Applications, San Diego, Aug. 4-9, (2007); see also: http://www.cs.berkeley.edu/ sequin/PAPERS/2007 SIGGRAPH 57Cell.pdf (accessed April 10, 2010).

[10] J. Smith, SLIDE design environment. (2003): http://www.cs.berkeley.edu/ ug/slide/ (accessed March 10, 2010). 